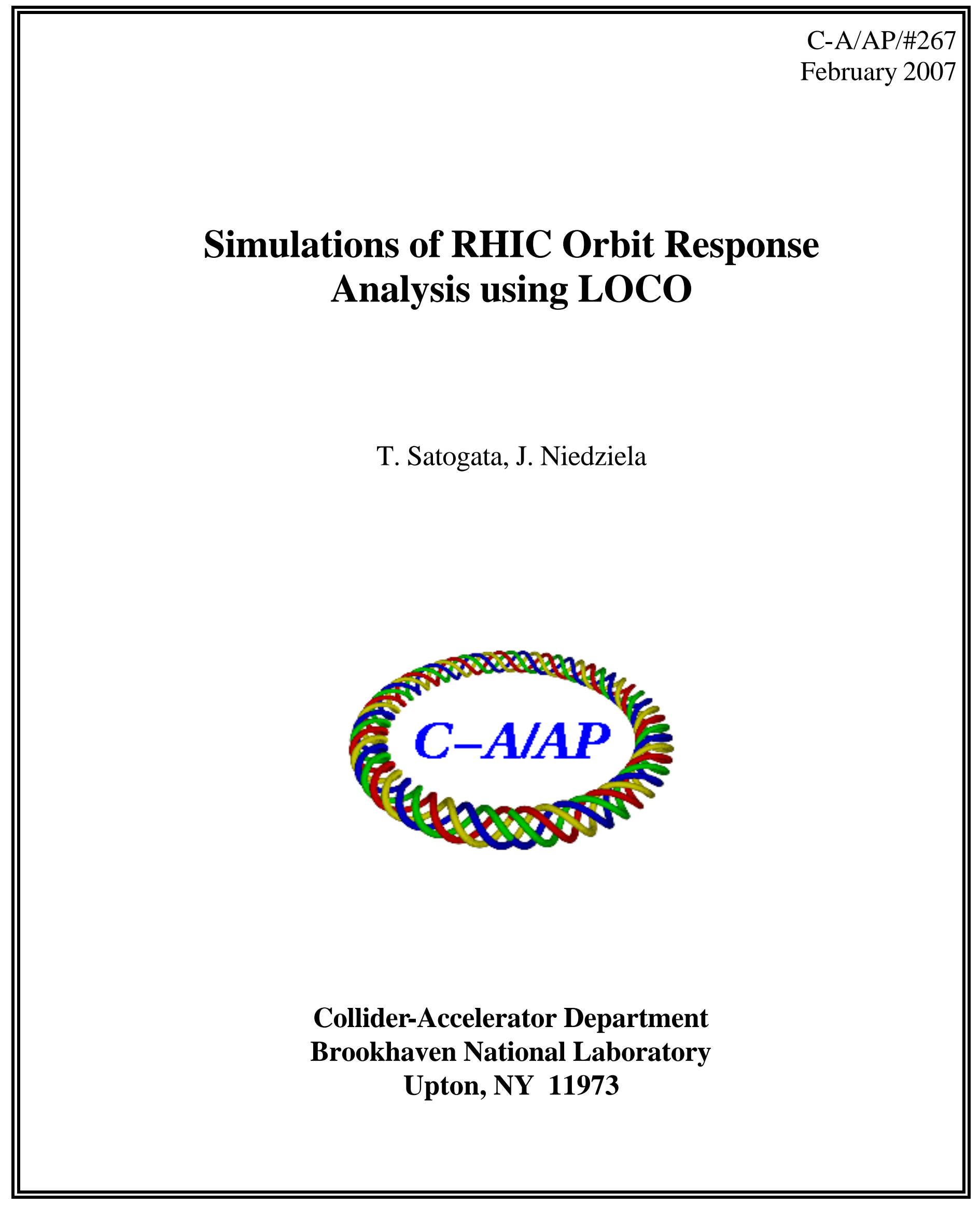




\title{
Simulations of RHIC Orbit Response Analysis using LOCO
}

\author{
T. Satogata and J. Niedziela
}

January 31, 2007

\begin{abstract}
Orbit response matrix (ORM) measurements are used in many circular accelerators to measure and correct gradient and optics errors, beam position monitor (BPM) gain errors, and dipole corrector gain errors [1]. Here we report results of numerical studies of the RHIC FY06 polarized proton injection and storage lattices to evaluate the feasibility of RHIC ORM analysis, and quantify the effect of random errors (including BPM noise) on ORM analysis of the RHIC lattice.
\end{abstract}

\section{ORM Description}

An orbit response matrix $(\mathrm{ORM}) \mathcal{R}$ is a matrix that associates BPM difference orbit changes $\Delta x_{i}$ with corrector changes $\Delta \theta_{j}$ :

$$
\left(\begin{array}{c}
\Delta x_{1} \\
\Delta x_{2} \\
\ldots \\
\Delta x_{n}
\end{array}\right)=\left(\begin{array}{cccc}
R_{11} & R_{12} & \ldots & R_{1 m} \\
R_{21} & R_{22} & \ldots & R_{2 m} \\
\ldots & \ldots & \ldots & \ldots \\
R_{n 1} & R_{n 2} & \ldots & R_{n m}
\end{array}\right)\left(\begin{array}{c}
\Delta \theta_{1} \\
\Delta \theta_{2} \\
\ldots \\
\Delta \theta_{n}
\end{array}\right) \equiv \mathcal{R}\left(\begin{array}{c}
\Delta \theta_{1} \\
\Delta \theta_{2} \\
\ldots \\
\Delta \theta_{n}
\end{array}\right)
$$

The ORM includes coupling when $(i, j)$ range over horizontal and vertical BPMs and correctors. $R_{i j}$ is well-known in a linear uncoupled lattice, but can also include nonlinear and coupling terms such as those from closed orbit sextupole feed-down, and dispersive terms due to path length changes.

The ORM can be modeled $\left(\mathcal{R}_{\text {model }}\right)$ and measured $\left(\mathcal{R}_{\text {meas }}\right)$. A nonzero difference $\mathcal{R}_{\text {diff }} \equiv \mathcal{R}_{\text {model }}-\mathcal{R}_{\text {meas }}$ is sensitive to many error sources including quadrupole gradient errors, quadrupole rolls, BPM gain errors, corrector gain errors, and sextupole closed orbit offsets. This sensitivity has been used at several accelerators to correct these errors, and bring machine optics much closer to modeled optics [1]. The model should be as complete a design model as possible so differences are attributable to errors in the actual machine and not shortcomings of the model.

In that spirit, the objective of ORM analysis is to minimize $\mathcal{R}_{\text {diff }}$ by varying machine parameters $v_{k}$. This is done by solving

$$
\left(\begin{array}{c}
\Delta R_{11} \\
\Delta R_{12} \\
\cdots \\
\Delta R_{n(m-1)} \\
\Delta R_{n m}
\end{array}\right)=\mathcal{J}_{\text {model }}\left(\begin{array}{c}
\Delta v_{1} \\
\Delta v_{2} \\
\cdots \\
\Delta v_{N-1} \\
\Delta v_{N}
\end{array}\right)
$$

where

$$
\mathcal{J}_{\text {model }} \equiv\left(\begin{array}{cccc}
\partial R_{11} / \partial v_{1} & \partial R_{11} / \partial v_{2} & \ldots & \partial R_{11} / \partial v_{N} \\
\partial R_{12} / \partial v_{1} & \partial R_{12} / \partial v_{2} & \ldots & \partial R_{12} / \partial v_{N} \\
\ldots & \ldots & \ldots & \ldots \\
\partial R_{n(m-1)} / \partial v_{1} & \partial R_{n(m-1)} / \partial v_{2} & \ldots & \partial R_{n(m-1)} / \partial v_{N} \\
\partial R_{n m} / \partial v_{1} & \partial R_{n m} / \partial v_{2} & \ldots & \partial R_{n m} / \partial v_{N}
\end{array}\right)
$$

Here $\Delta R_{i j} \equiv R_{i j \text {,model }}-R_{i j \text {,meas }}$ (be careful of the sign), and $v_{k}$ are the fit variables such as quadrupole gradient error, quadrupole roll, etc. The differentials $\partial R_{i j} / \partial v_{k}$ in the large response matrix Jacobian $\mathcal{J}_{\text {model }}$ are obtained from the model, either from variational principles or modeled response matrices. These terms are trivial to generate for BPM and corrector gain errors.

The ORM solution is usually improved by weighting Eqn. (2) with measured BPM noise levels $\sigma_{i}$. This is accomplished by dividing both $\Delta R_{i j}$ and $\partial R_{i j} / \partial v_{k}$ by $\sigma_{i}$ before solving. For simplicity, we avoid a notation 
change and assume that this weighting is applied in the remainder of this paper; this becomes important when BPM noise is introduced in Sections 5 and 6.

$\mathcal{J}_{\text {model }}$ is usually not square, and the ORM problem for accelerators is always overconstrained with $n \times m$ (the number of BPMs times the number of correctors) being much greater than $N$ (the number of fit variables). The least-squares solution to Eqn. (2) is most easily found with singular value decomposition (SVD) [2].

$$
\mathcal{J}_{\text {model }}=\mathcal{U S} \mathcal{V}^{\mathrm{T}}
$$

where $\mathcal{U}$ is an $n m \times N$ orthogonal matrix, $\mathcal{V}$ is an $N \times N$ orthogonal square matrix,

$$
\mathcal{U} \mathcal{U}^{\mathrm{T}}=\mathcal{V} \mathcal{V}^{\mathrm{T}}=\mathcal{I}
$$

and $\mathcal{S}$ is an $N \times N$ diagonal matrix whose elements are the singular values of $\mathcal{J}_{\text {model }}$. Using Eqn. (5),

$$
\mathcal{J}_{\text {model }}{ }^{-1}=\mathcal{V} \mathcal{S}^{-1} \mathcal{U}^{\mathrm{T}}
$$

In practice very small singular values (corresponding to degeneracies of the parameter space) are eliminated by setting the corresponding diagonal elements of $\mathcal{S}^{-1}$ to zero. "Very small" depends on the problem, and one objective of this work is to determine the appropriate singular value cut for the RHIC lattice, so degeneracies are properly removed without ruining the effectiveness of the SVD solution given below.

The optimal least-squares solution for Eqn. (2) is then given [3] by

$$
\left(\begin{array}{c}
\Delta v_{1} \\
\Delta v_{2} \\
\ldots \\
\Delta v_{N-1} \\
\Delta v_{N}
\end{array}\right)=\mathcal{J}_{\text {model }}{ }^{-1}\left(\begin{array}{c}
\Delta R_{11} \\
\Delta R_{12} \\
\ldots \\
\Delta R_{n(m-1)} \\
\Delta R_{n m}
\end{array}\right)=\mathcal{V S}^{-1} \mathcal{U}^{\mathrm{T}}\left(\begin{array}{c}
\Delta R_{11} \\
\Delta R_{12} \\
\ldots \\
\Delta R_{n(m-1)} \\
\Delta R_{n m}
\end{array}\right)
$$

Since the dependence of $\Delta R_{i j}$ is not linear on some variables $\Delta v_{k}$ (such as quadrupole gradients), each solution should be applied to the model and iterated (with $\mathcal{J}_{\text {model }}$ changing with every iteration) until the solution for $\Delta v_{k}$ converges. Rather than write separate analysis software, we have chosen to use the LOCO (Linear Optics from Closed Orbits [4]) and AT (Accelerator Toolkit [5]) packages in a Matlab environment to evaluate the feasibility of ORM analysis and gradient corrections in RHIC.

\section{Matlab, AT, and LOCO Infrastructure}

Matlab v7 is installed for user mcr on the host cheetah.pbn.bnl.gov behind the C-AD operations firewall, in the directory /home/cheetah/matlab. This license permits any number of concurrent sessions as long as they are only executed by the mcr account on this host. The mcr Matlab working area is located in the directory

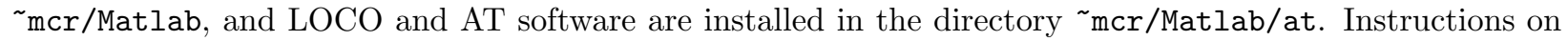
how to set up this Matlab environment to use AT and LOCO are in the file mcr/Matlab/README.

To evaluate RHIC lattices in this environment, they must be converted to the Matlab AT language. We have written a perl script, RhicSxfToAT.pl, to convert RHIC SXF lattices acquired from the RHIC online model using RhicModelPlayer to AT. The resulting Matlab AT lattice file puts the RHIC lattice in the standard AT global variable THERING. The conversion process is straightforward, but a few items are of interest:

- All quadrupoles are modeled with the StrMPoleSymplectic4Pass element model [5]. Changes to quadrupole strengths in the lattice are effected by changing PolynomB(2) of the given quadrupole instead of the $\mathrm{K}$ value. This element model was chosen so skew and kick elements within quadrupoles can be controlled independently. Calculations do not substantially differ from those using the simpler QuadLinearPass element model.

- The RHIC lattice includes thin focusing kicks at the outside ends of the separation DX dipole magnets, where the design orbit is offset from the magnet center. These focusing kicks are modeled as multipoles in the RHIC online model SXF lattice, and are modeled as additional thin multipoles in the AT lattice.

Comparison of optics functions between the AT and MADX models for several RHIC low- $\beta$ storage lattices shows differences of less than $0.1 \%$. This difference is negligible for the purposes of ORM analysis and LOCO where we are correcting $\beta$ errors of $1-20 \%$. 
The RHIC lattices considered in this study were converted from the RHIC online model for the Yellow ring, pp35: : injection, on May 11 2006, and pp35: : store on May 30 2006. These lattices correspond to ORM measurements acquired on those dates. Analysis of these measurements will be reported in another paper. These lattices are referred to in this paper as the RHIC injection and store lattices. All Matlab files

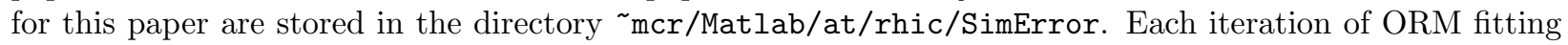
with all BPMs, correctors, and quadrupoles took approximately 30 minutes on cheetah.

The ORMs for this paper are all generated with dipole corrector changes of $20 \mu \mathrm{rad}$. This dipole kick in an arc dipole corrector produces a maximal arc orbit change of $3 \mathrm{~mm}$, and up to $8-10 \mathrm{~mm}$ in the low- $\beta$ triplets. These are the dipole corrector changes that were used in the 2006 RHIC ORM measurements.

\section{Initial Testing: A Small Set of Large Errors}

For an initial test, we introduce four $5 \%$ quadrupole errors in the RHIC yellow injection lattice: yi6-qd16 by $-0.0043 \mathrm{~m}^{-2}$, yi7-qf9 by $+0.0040 \mathrm{~m}^{-2}$, yi2-qd10 by $+0.0043 \mathrm{~m}^{-2}$, and yi3-qf3 by $-0.00273 \mathrm{~m}^{-2}$. These errors are much larger than realistic physical errors in these quadrupoles; fortunately the injection lattice is quite forgiving, and these errors only create a $5-10 \%$ beta wave in both planes of RHIC. Data for this example is in Matlab file locoTest.m. All quadrupoles, BPMs, and correctors in the lattice are used, and there are no noise or gain errors in the BPMs and correctors.

The figure of merit for the difference between modeled (or ideal) and measured (or simulated error) orbit response matrices in LOCO is $\chi^{2} /$ dof. This is typically close to 0 for an excellent fit, and $\gg 1$ for bad fits. With the above errors in a simulated test lattice, the initial $\chi^{2} /$ dof $=11253$; the difference between ideal and simulated ORMs ranges over $0.5 \mathrm{~mm}$. After two iterations of LOCO, fitting all quadrupole gradients, this reduces to 10, and the residual error between the ideal and simulated ORMs is less than $15 \mu \mathrm{m}$ at all BPMs. The four large quadrupole errors are correctly fitted. The final ORM difference, singular values from ORM analysis, and quadrupole fits are shown in Fig. 1. Similar tests for randomly chosen $5 \%$ quadrupole errors also converge in two iterations for blue and yellow storage lattices.
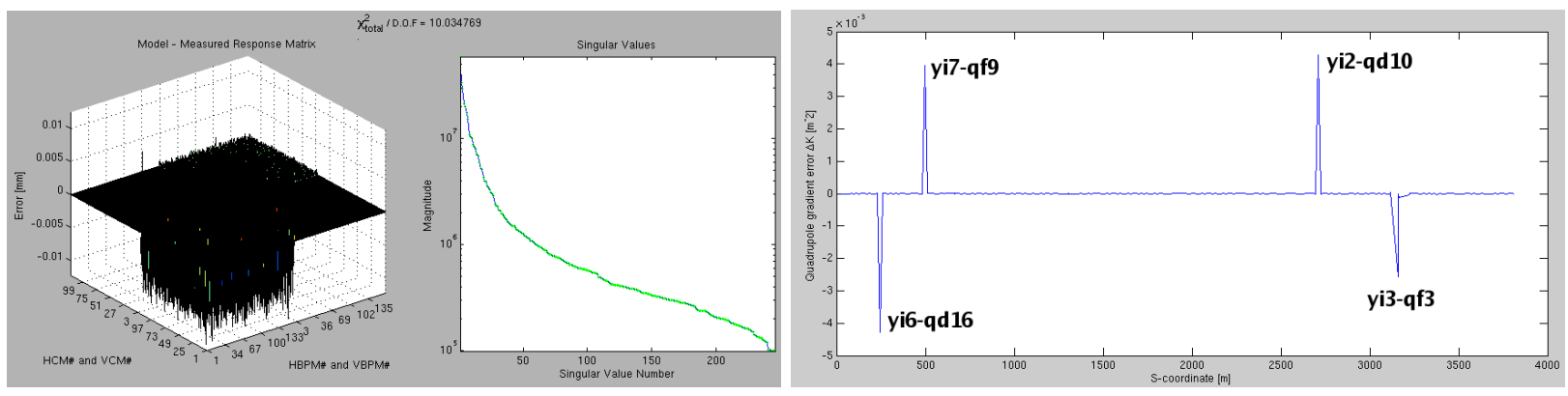

Figure 1: Difference between model and measured ORM after convergence, singular values, and quadrupole fits for a test of ORM with the yellow injection lattice. Four artificial large errors were introduced, with perfect BPMs and correctors; LOCO analysis correctly determines these errors in two iterations.

\section{Random Quadrupole Errors with Perfect BPMs}

The previous section demonstrated that LOCO can find a small set of large errors, but $5 \%$ gradient errors are much larger than the $0.1-0.2 \%$ gradient errors typically expected in RHIC. LOCO has been used to find errors of this magnitude at the NSLS X-Ray ring [1]. To evaluate whether LOCO can find similar errors in the ideal RHIC lattice, we simulated uniformly distributed random errors ranging over $\pm 0.1 \%$ in the yellow injection and store lattices, and compared errors fitted by LOCO analysis to the simulated errors. The difference between model and measured ORMs is $100-500 \mu \mathrm{m}$, or $5-10 \%$ of the closed orbit changes during ORM measurement.

For the $0.1 \%$ yellow storage lattice case, LOCO rapidly converges from $\chi^{2} / \mathrm{dof}=2757$ to $10^{-6}$ in three iterations, and all quadrupole errors are fitted to better than $1 \sigma$ of the LOCO fit error bars (see Fig. 2). This analysis uses all singular values in the LOCO SVD analysis, and fits all quadrupoles in RHIC. The $0.1 \%$ yellow storage lattice error case is in the Matlab file locoyellowStore0p1ideal.m. Simular results are 


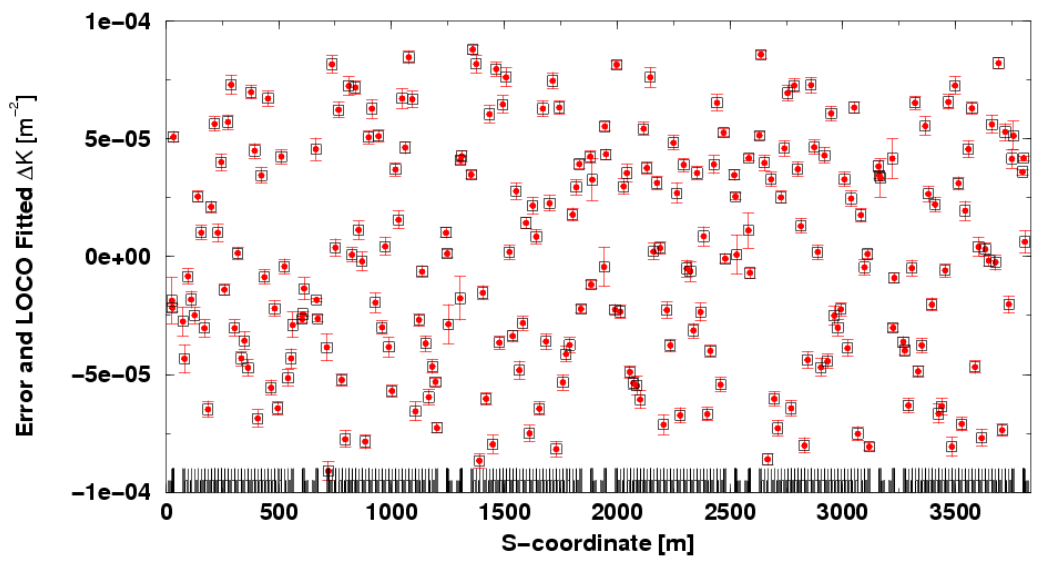

Figure 2: $0.1 \%$ gradient error values in the yellow storage lattice (black squares) and values fit by LOCO in three iterations (red circles and error bars) with perfect BPMs.

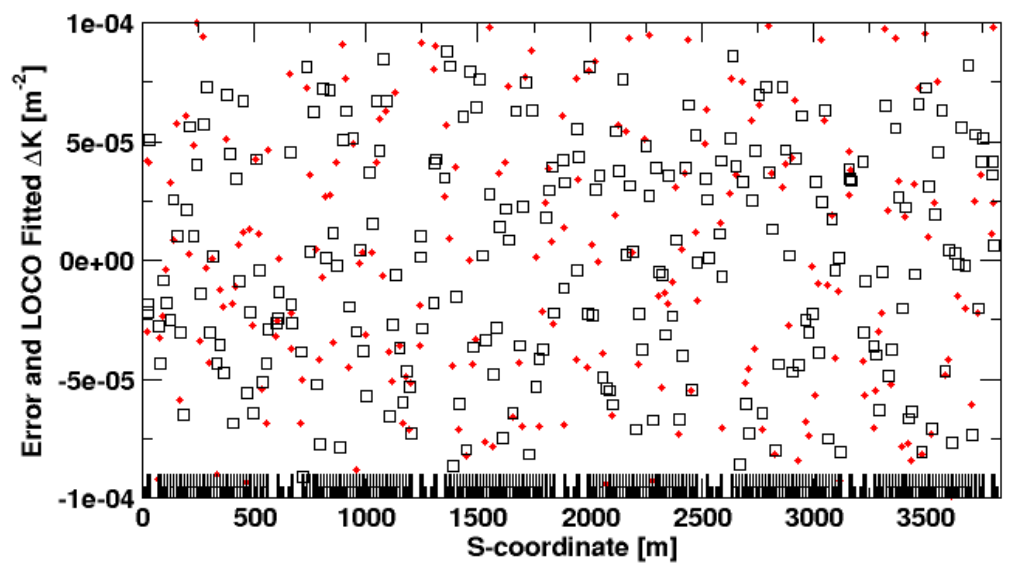

Figure 3: $0.1 \%$ gradient error values in the yellow storage lattice (black squares) and values fit by LOCO in three iterations (red dots) with $30 \mu \mathrm{m}$ random BPM noise. Error bars for the fits are not shown, as they are $2-3 \times 10^{-4} \mathrm{~m}^{-1}$, larger than the vertical axis size.
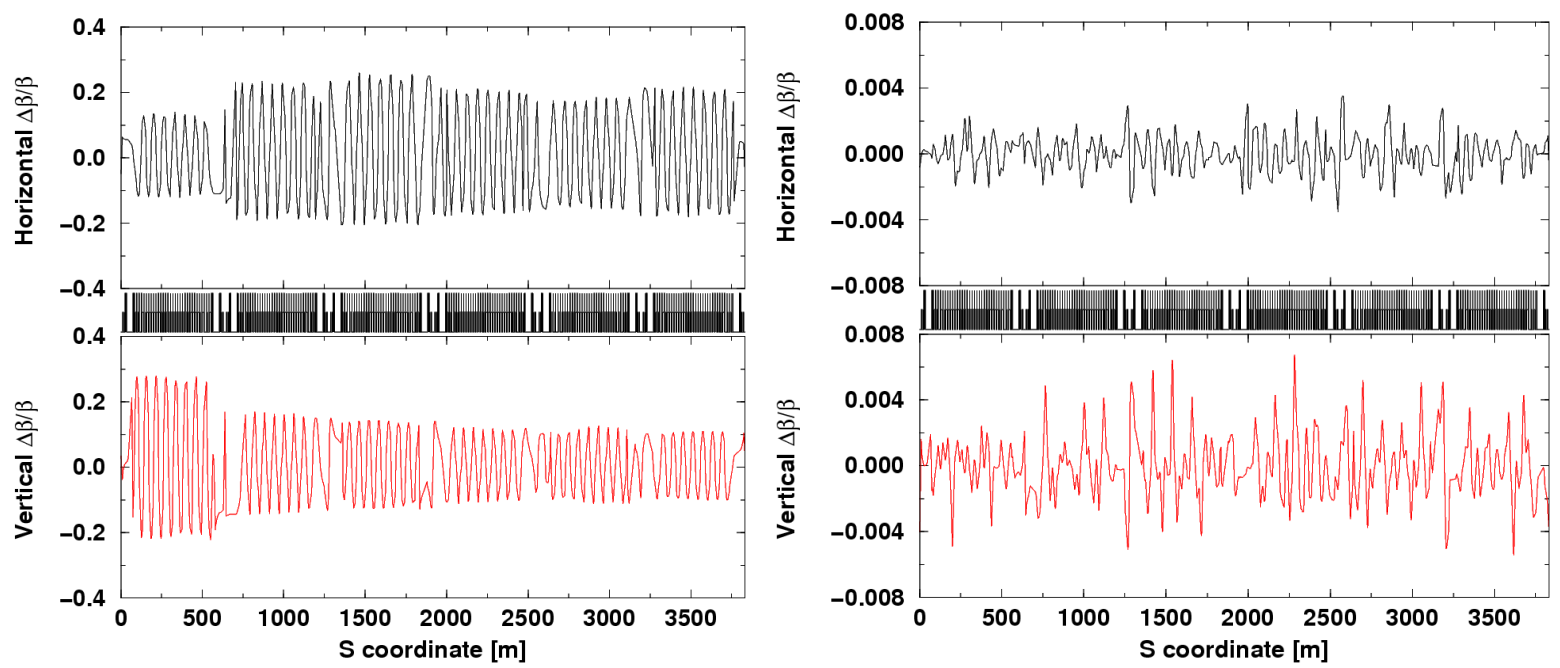

Figure 4: The effects of flat-distribution random $0.1 \%$ gradient errors on the beta functions of the yellow storage lattice, showing $\pm 20 \%$ beta waves (left), and residual beta function error between the model and LOCO fitting with $30 \mu \mathrm{m}$ random BPM noise (right). 
found for the injection and blue storage lattices. This indicates that the RHIC lattice is not problematically degenerate for purposes of orbit response and LOCO analysis.

\section{$5 \quad$ Effects and Limits of BPM Noise}

Existing ORM data uses the RHIC BPM average orbit system, which averages 10,000 consecutive turns of position data to provide an average orbit. In a typical arc BPM with $\beta \approx 50 \mathrm{~m}$, measured average orbit RMS noise is $15 \mu \mathrm{m}$, split equally between intrinsic noise and random bias from $10 \mathrm{~Hz}$ beam motion. The $10 \mathrm{~Hz}$ bias will be minimized in the future by changing the BPM averaging period to a multiple of 7,800 turns. The short-term BPM RMS average orbit noise is $7 \mu \mathrm{m}$ in store conditions with a 7,800 turn averaging period [6].

We repeated the $0.1 \%$ yellow storage lattice case from the previous section with a conservative $30 \mu \mathrm{m}$ of random noise on all BPM measurements. This is 7-20\% of the "signal", or difference between modeled and measured ORMs for LOCO analysis. Three iterations of LOCO converged from $\chi^{2} / \mathrm{dof}=3.4$ in the first iteration to $\chi^{2} /$ dof $=0.34$ in the last. Further iterations did not improve $\chi^{2} /$ dof, as the remaining ORM differences between the measurement and model were completely attributable to noise.

The simulated quadrupole errors and values fit by LOCO are shown in Fig. 3. Comparison to the case without BPM noise (Fig. 2) shows no strong correlation between simulated and fitted quadrupole errors. However, the error bars from LOCO analysis are larger than the scatter of simulated errors; in this sense the LOCO fit is good. The LOCO fitted gradient errors also reproduce the optics errors through the ring, improving beta beating by a factor of about 100 as shown in Fig. 4. With this relative BPM accuracy, the optics in RHIC can be accurately modeled even without absolutely correct fits of the simulated gradient errors.

Fig. 5 shows LOCO fits of the same $0.1 \%$ gradient errors with $5 \mu \mathrm{m}$ of random noise on all BPM measurements, $1-3 \%$ of the difference between modeled and measured ORMs. All gradient errors are within $1 \sigma$ of the LOCO fit. This level of relative BPM accuracy is required if the objective of ORM is to measure gradient errors accurately for the RHIC storage lattice.

It is important to note that the relative BPM error depends on the difference between the modeled and measured ORMs; this difference scales linearly with the dipole corrector strengths used to excite difference orbits for the ORM meaurement. Large dipole corrector strengths induce sextupole feed-down and risk aperture-induced beam loss, while small dipole corrector strengths make the relative BPM errors large. The best approach is typically to settle on a desired difference orbit amplitude, and scale all corrector strengths by $\sqrt{\beta}$ to produce difference orbits of this amplitude for each corrector. The LOCO package can properly analyze this case, when the excitations of correctors are nonuniform.

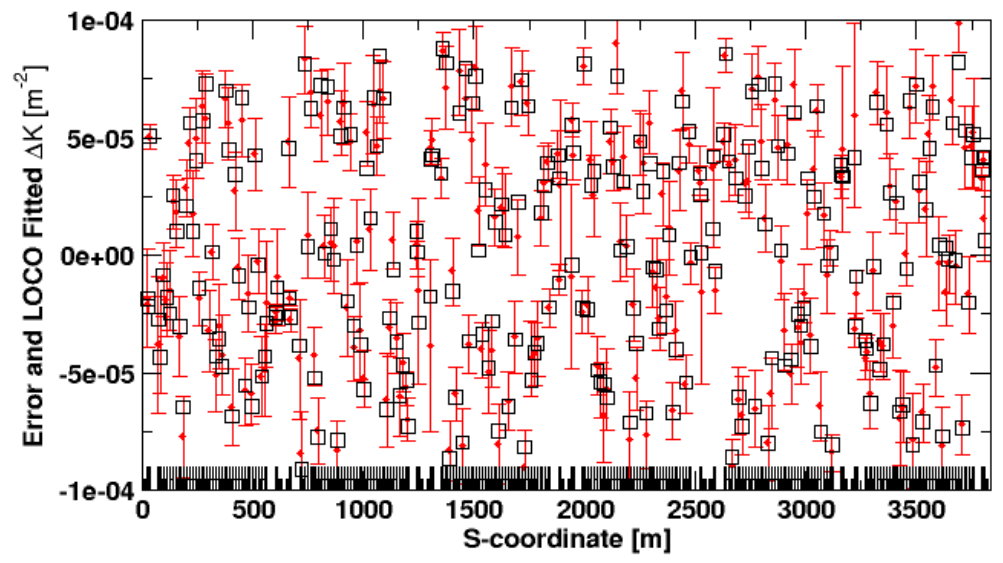

Figure 5: $0.1 \%$ gradient error values in the yellow storage lattice (black squares) and values fit by LOCO in three iterations (red dots) with $5 \mu \mathrm{m}$ random BPM noise. Correlation between fitted and actual errors is high, and all gradient errors are within $1 \sigma$ of the fit. 


\section{Effects and Limits of BPM/Corrector Gain Errors}

Initial attempts to fit RHIC BPM and corrector gain errors with Matlab LOCO diverged, even when gradient errors were fitted first. Introducing a harder singular value cut (using only singular values above $10^{-5}$ of the maximum singular value) resolved this problem; this cut removed two obvious scaling degeneracies introduced by fitting all BPM and corrector gains. This permits LOCO analysis of realistic RHIC ORM data, including $\mathrm{BPM}$ and corrector gain errors.

To test these gain errors, BPM and corrector gain errors were randomly distributed over $\pm 5 \%$ and applied to the simulation conditions of the previous section (yellow store lattice, $\pm 0.1 \%$ random gradient errors), and these errors were fitted with LOCO as extra degrees of freedom along with gradient errors. Source code for this test is located in the file locoYellowStore0p1_bpmcorr5percent. dat. This LOCO fit converged to fit BPM and corrector gains and gradient errors nearly perfectly in 3 iterations, reducing $\chi^{2} /$ dof from 3.6 to $10^{-4}$.

Fig. 6 shows BPM and corrector gain error data from this test. The LOCO fit and original model errors agree to within $1 \sigma$ for nearly all BPMs and correctors, though there are discrepancies in the 5 o'clock area, particularly for corrector gains. This is attributable to the high beta functions in IR6 triplets, and can be addressed with judicious additional singular value cuts in the LOCO analysis.
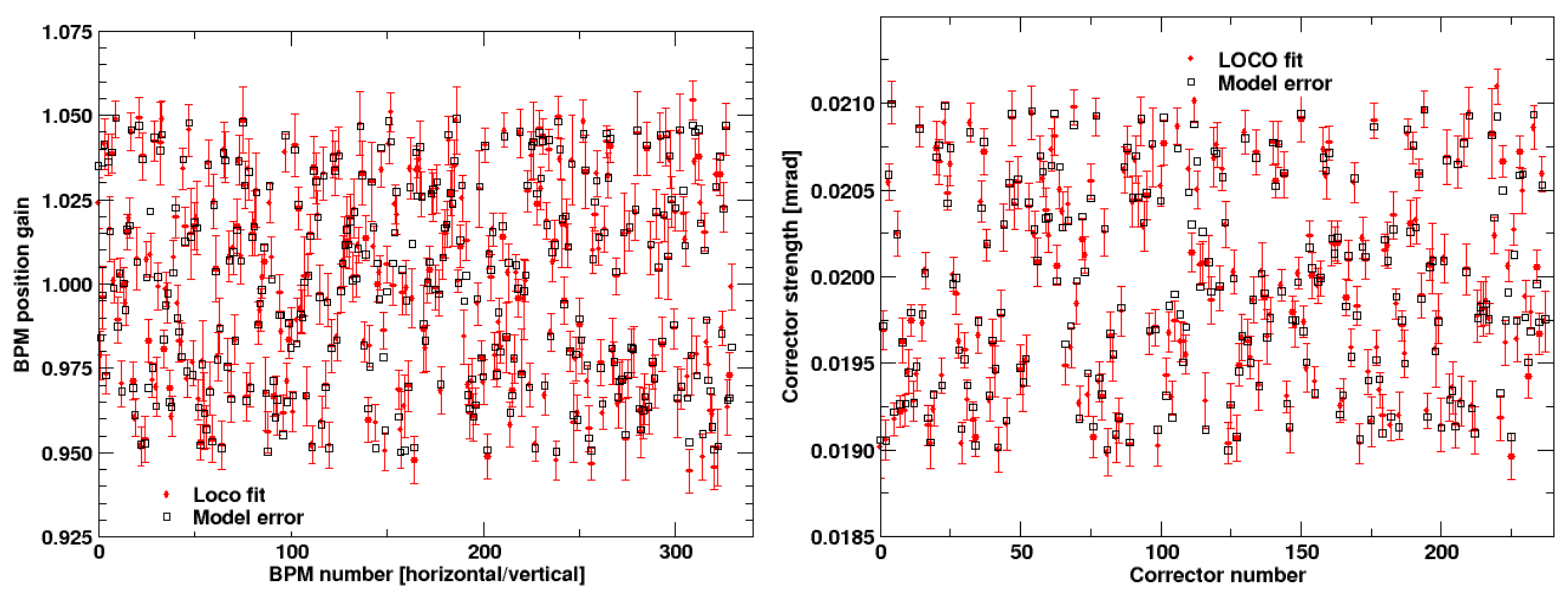

Figure 6: Modeled random errors and LOCO fits for BPM and corrector gain errors in the yellow storage lattice, with no BPM noise. This model generated $\pm 5 \%$ gain errors for BPMs and correctors, and fitted $\pm 0.1 \%$ quadrupole errors. Quadrupole error fits were comparable to Fig.2

Table 1: RMS spread around a perfect spread for LOCO fits of random $\pm 5 \% \mathrm{BPM}$ and corrector gain errors, with random $\pm 0.1 \%$ quadrupole gradient errors, in the yellow store lattice. Raw data for the $30 \mu \mathrm{m}$ noise case is shown in Fig. 7 .

\begin{tabular}{|c|c|c|}
\hline BPM Noise & $\begin{array}{c}\text { BPM gain fit } \\
\text { rms spread }\end{array}$ & $\begin{array}{c}\text { Corrector error fit } \\
\text { rms spread }[\mathrm{mrad}]\end{array}$ \\
\hline No noise & $2.7 \times 10^{-3}$ & $4.5 \times 10^{-5}$ \\
$5 \mu \mathrm{m}$ noise & $4.2 \times 10^{-3}$ & $4.3 \times 10^{-5}$ \\
$30 \mu \mathrm{m}$ noise & $3.9 \times 10^{-3}$ & $6.4 \times 10^{-5}$ \\
\hline
\end{tabular}

Can BPM and corrector gain errors of this magnitude be determined using LOCO with realistic BPM errors? We consider the same two cases (30 $\mu \mathrm{m}$ and $5 \mu \mathrm{m}$ BPM noise) as the previous section. ORM analysis of these cases converges quickly and reduces beta beating by a factor of 200-300, consistent with Fig.4.

Fig. 7 compares model errors and LOCO fits of those model errors in the yellow storage lattice, with $30 \mu \mathrm{m}$ BPM noise, $\pm 5 \%$ random BPM and corrector gain errors, and $\pm 0.1 \%$ quadrupole gradient errors. Even with realistic noise, BPM and corrector gain errors are within $1 \sigma$ of the fit produced by LOCO. The RMS spreads around a perfect fit for all cases are listed in Table 1. In the cases of no BPM noise and $30 \mu \mathrm{m}$ BPM noise, scaled error spreads are comparable for BPM and corrector gain errors, and are less than 
0.5\%. These BPM and corrector gain uncertainties from LOCO analysis are achievable with realistic BPM noise.
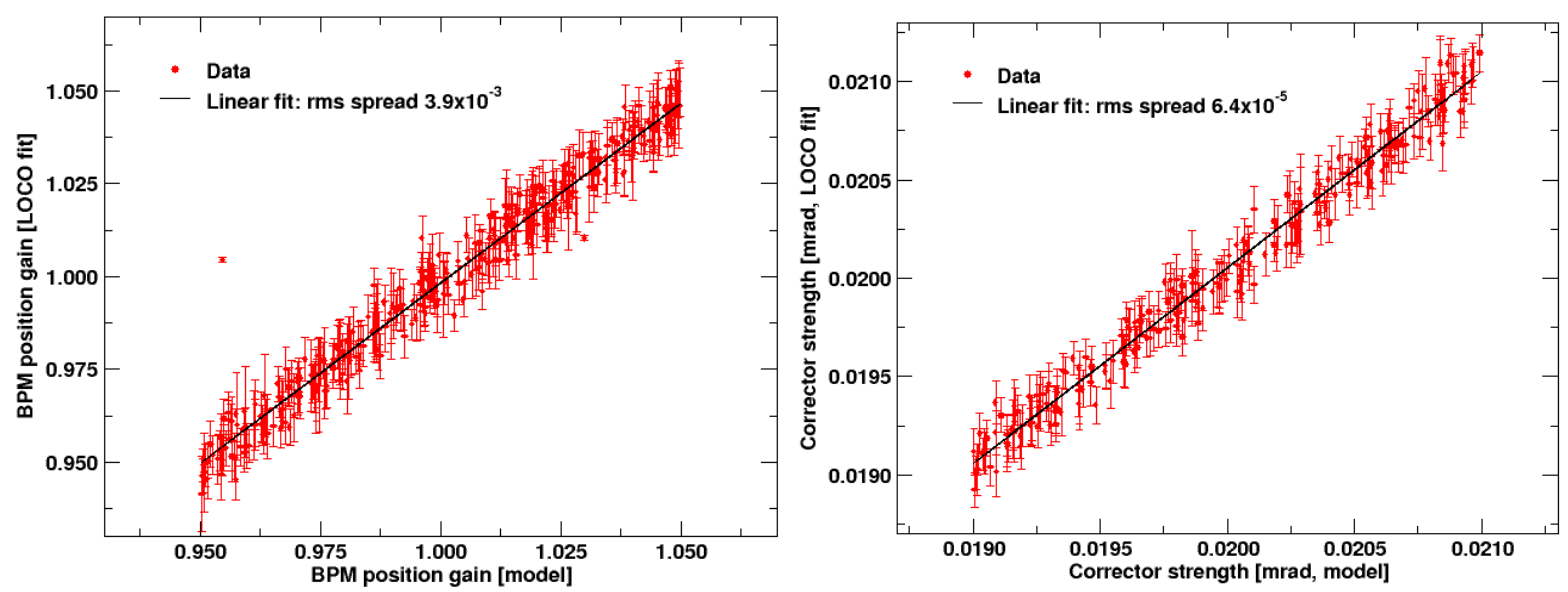

Figure 7: Modeled random errors and LOCO fits for BPM and corrector gain errors in the yellow storage lattice, with $30 \mu \mathrm{m}$ BPM noise. LOCO accurately finds nearly all BPM and corrector gain errors with this level of noise, though there are occasional outliers; for example, the BPM outlier is yo7-bv3, a vertical BPM located in the 7 o'clock low-beta region.

\section{Conclusions}

An infrastructure to analyze orbit response matrices (ORMs) using Matlab LOCO and AT packages has been installed in C-AD. With no noise in RHIC BPMs, simulated $0.1 \%$ gradient errors in the Run-6 pp yellow storage lattice were fitted perfectly by LOCO; this lattice is not degenerate for the purposes of fitting all quadrupoles for gradient errors with ORM and LOCO analysis.

RHIC average orbit BPM noise is about $30 \mu \mathrm{m}$ RMS in arc BPMs. With ORM corrector strengths of $20 \mu \mathrm{rad}$ and this level of BPM noise, $0.1 \%$ gradient errors cannot be individually resolved, but beta beating is still reduced by two orders of magnitude. Improvement of orbit measurements to $5 \mu$ m RS lowers gradient fit error bars to about $0.02 \%$ of set gradient. ORM analysis of BPM and corrector gain errors in the presence of realistic $30 \mu \mathrm{m}$ BPM noise produces correct gains to an accuracy of much less than $1 \%$, though care must be taken with low- $\beta$ IR triplet correctors and BPMs due to their high sensitivity.

\section{References}

[1] J. Safranek, "Experimental Determination of Storage Ring Optics Using Orbit Response Measurements", NIM in Physics Research A 388 (1997) pp. 27-36.

[2] http://mathworld.wolfram.com/SingularValueDecomposition.html

[3] W. Press et al., "Numerical Recipes in C" (Second Edition), 1992, pp. 676-9.

[4] J. Safranek, G. Portmann, A. Terebilo, C. Steier, "Matlab-Based LOCO", European Particle Accelerator Conference, Paris, France, June 2002.

[5] A. Terebilo, "Accelerator Toolbox for Matlab", SLAC-PUB 8732, May 2001, and http://ww-ssrl.slac.stanford.edu/at.

[6] BPM_2006 elog, entry dated June 15 2006, 14:11. 